\title{
Historia del Programa de Control de la Tuberculosis de Chile
}

\author{
TANIA HERRERA M.* y VICTORINO FARGA C.**
}

\section{History of the Program of Control of Tuberculosis in Chile}

A book on the history of the Program of Control of Tuberculosis in Chile, recently published by the Ministry of Health as a landmark of the Public Health of the country, is commented. His author, the distinguished historian Marcelo López Campillay goes over the different stages of the tuberculosis epidemic in Chile and the successive attempts to control it. First with a modern control program and later with a program aimed at the elimination of tuberculosis as a public health problem, with a target of less than 5 cases per 100,000 inhabitants by 2020. The successes and difficulties of the program through its different stages are explained by the testimonies from its main protagonists and conductors. Complementary contributions about this exciting history are given.

Key words: Tuberculosis in Chile, tuberculosis control \& elimination program.

\section{Resumen}

Se comenta la publicación del Ministerio de Salud sobre la historia del Programa de Control de la Tuberculosis, como un hito dentro de la Salud Pública de Chile. Su autor, el distinguido historiador Marcelo López Campillay, recorre las distintas etapas vividas por la epidemia de tuberculosis en Chile y los sucesivos intentos de controlarla a través de un Programa de Control de la Tuberculosis moderno, que ha ido evolucionando a un programa cuya meta es la eliminación de la enfermedad como problema de salud pública, con una meta de menos de 5 casos por 100.000 habitantes para el año 2020. Se detallan los éxitos y dificultades que ha tenido que enfrentar el programa en sus distintas etapas, basados en los testimonios de sus principales protagonistas y se agregan aportes complementarios sobre esta interesante historia.

Palabras clave: Tuberculosis en Chile, Programa de control y eliminación.

El Ministerio de Salud ha tenido la feliz idea de publicar una Serie de "Hitos de la Salud Pública en Chile" y como primer ejemplo ha elegido la Historia del Programa de Control de la Tuberculosis (PCT). Es así como la Unidad de Patrimonio Cultural de la Salud, de la División de Planificación Sanitaria del Ministerio de Salud, acaba de publicar "Medicina, política y bien común: 40 años de historia del programa de control de la tuberculosis (1973-2013)", cuyo autor es el distinguido historiador Marcelo López Campillay, investigador del Programa de Estudios Médicos
Humanísticos de la Facultad de Medicina de la Pontificia Universidad Católica de Chile ${ }^{1}$.

El método de trabajo del Sr. López, además de la revisión de la extensa bibliografía existente sobre la tuberculosis en Chile, se ha basado en buena parte en largas entrevistas a los principales protagonistas de esta historia. A continuación se describen los principales hitos en la historia del PCT en Chile, a modo de incentivar la lectura del libro señalado y también como una forma de ofrecer una visión propia de lo que ha sido la evolución de este programa de salud pública.

\footnotetext{
* Directora del Programa de Control y Eliminación de la Tuberculosis, Ministerio de Salud.

** Médico asesor del Ministerio de Salud.
} 


\section{La tuberculosis en Chile en la era pre-quimioterapia}

Hasta mediados del siglo XIX la tuberculosis era relativamente infrecuente en Chile y se manifestaba principalmente como escrofulosis, forma de tuberculosis ganglionar debida al Mycobacterium bovis trasmitido por la leche de vaca antes de su pasteurización obligatoria.

Durante la segunda mitad del siglo XIX, en pleno desarrollo de la llamada Revolución Industrial, la tuberculosis alcanzó en Chile los niveles de una epidemia, manifestada inicialmente por altos índices de mortalidad. En esa época, la causa de la enfermedad se atribuía a factores sociales, porque claramente atacaba con más fuerza a la población más desvalida.

A la elevada mortalidad sucedió una creciente morbilidad y es así como a fines del siglo XIX empezaron los primeros intentos de "lucha contra la tuberculosis", que inicialmente se concentraron en la construcción de sanatorios: El Peral, San José de Maipo, Valparaíso, Putaendo, Peñablanca, entre otros. También aparecieron los primeros esbozos de organizaciones de beneficencia, como los llamados Dispensarios y Ligas antituberculosas.

Durante la primera mitad del siglo XX hizo su aparición una nueva especialidad médica: la Tisiología, que fue consolidada por el Profesor Héctor Orrego Puelma quien en rápida sucesión, a partir de 1930, creó la Sociedad Chilena de Tisiología, la Revista Aparato Respiratorio y Tuberculosis y, a continuación, ocupó la recientemente creada Cátedra de Tisiología en la Facultad de Medicina de la Universidad de Chile 2 . Poco a poco los gobiernos fueron tomando conciencia de la gravedad del problema de la tuberculosis y crearon la Ley de Medicina Preventiva, enfocada a la detección temprana de la enfermedad y las Secciones de Tisiología en las grandes organizaciones médicas de la época, la Dirección General de Beneficencia y la Caja del Seguro Obligatorio.

\section{La tuberculosis en Chile en la era de la quimioterapia}

En esta etapa se fueron sucediendo una serie de avances que permitieron un enfoque más científico para enfrentar la nueva epidemia. Primero el descubrimiento de la etiología infecciosa de la tuberculosis por Robert Koch en 1882, que convirtió a la bacteriología en el paradigma del diagnóstico y control de la "peste blanca"; luego la introducción, durante la primera mitad del siglo
$\mathrm{XX}$, de la vacuna BCG que tantas vidas de niños ha salvado y, por fin, el descubrimiento de los primeros fármacos efectivos contra la enfermedad a mediados del siglo pasado y, desde entonces, la sucesiva implementación de programas de control cada vez más eficaces.

El advenimiento de la quimioterapia antituberculosa encontró a Chile en un momento crucial de la historia de la medicina, como fue la creación del Servicio Nacional de Salud, lo que facilitó la aplicación de los avances científicos con amplia cobertura a lo largo de todo el país ${ }^{3}$.

La era moderna de la quimioterapia de la tuberculosis en Chile fue iniciada por un pequeño grupo de médicos del Hospital San Juan de Dios que descubrieron la importancia de administrar el tratamiento de la tuberculosis bajo observación directa, lo que se denominaba "tratamiento controlado" y permitía mejorar los resultados de la quimioterapia, asegurando la supervisión en la administración de las drogas empleadas y evitando el desarrollo de la temida resistencia bacteriana ${ }^{4}$. Rápidamente la nueva tecnología pudo aplicarse en amplia escala en todos los hospitales y consultorios del país, que se llenaron de "Centros de Tratamiento Controlado" y se entró en la era del tratamiento moderno de la enfermedad. Simultáneamente, ya se había reemplazado la pesquisa radiológica de la tuberculosis pulmonar mediante la abreugrafía, por la bacteriología con la "localización de casos" a través de la baciloscopia de la expectoración de los "sintomáticos respiratorios" consultantes espontáneos de los servicios de salud de todo el país. Es así como el Dr. Luis Herrera Malmsten desde el Instituto de Salud Pública creó una Red Nacional de Laboratorios de Bacteriología de la Tuberculosis que ha dado un sólido respaldo a los programas de diagnóstico y tratamiento ${ }^{5}$.

\section{La tuberculosis en la era del PROCET}

Un avance fundamental en la concepción del programa fue la iniciativa del Dr. Manuel Zúñiga, jefe del Programa de Control de la Tuberculosis (PCT) de la época, de fijar plazos concretos para la eliminación de la tuberculosis como problema de salud pública en Chile, creando el concepto de Programa de Control y Eliminación de la Tuberculosis (PROCET) y fijando una incidencia de 5 casos por 100.000 habitantes como meta para el año $2020^{6}$. Aunque de los plazos intermedios, sólo se cumplió el primero el año 2000, cuando la incidencia de la tuberculosis en todas sus formas estuvo por debajo de 20/100.000, el objetivo 
fijado para el año 2020 aún es alcanzable si se lograra optimizar las acciones del programa.

Durante todo el período, junto con seguir perfeccionando las Normas del PCT, se efectuaron importantes investigaciones operacionales. Destacan por su originalidad las que tuvieron por objeto encontrar mejores asociaciones de drogas antituberculosas, a través de estudios cooperativos en los cuales intervinieron todas las áreas de salud de Santiago, con la inapreciable ayuda de las enfermeras del programa. Así se llegó al Esquema TA-81 que prontamente reemplazó a los tratamientos en uso y ha tenido un notable impacto internacional ${ }^{7}$. También resulta destacable que, aún en tiempos difíciles, se haya podido efectuar casi todos estos años un "Curso Clínico-Epidemiológico de Tuberculosis", para médicos, enfermeras, y profesionales de laboratorio de Chile y Latinoamérica, que ha permitido mantener una masa crítica de profesionales expertos en cumplir las normas del PCT. La experiencia adquirida en estos cursos sirvió de base para la publicación del libro "Tuberculosis", cuyas segunda y tercera ediciones fueron incorporadas por la Organización Panamericana de la Salud dentro de su Programa de Textos y repartidas por toda América Latina ${ }^{8}$. Por otra parte, el PCT tuvo la fortuna, desde sus inicios, de contar con la asesoría de una serie de expertos internacionales entre los cuales se destacan el Profesor Georges Canetti en Bacteriología y al experto en Epidemiología y Programas de Control de la Tuberculosis Karel Styblo ${ }^{9}$. Igualmente afortunado fue contar con una sucesión de Jefes de Programa, en el Nivel Central del Ministerio de Salud, de excepcional calidad y dedicación. Durante este largo período, de más de 60 años, también ha funcionado con escasas interrupciones, un Comité Regional de Tuberculosis al que asisten regularmente médicos, enfermeras y laboratoristas de todas las áreas de Santiago, prestos a solucionar los múltiples problemas que se van presentando. Es evidente que todos estos factores contribuyeron a la mantención y perfeccionamiento del PCT a través de los años.

\section{Los desafíos actuales del PROCET}

La situación epidemiológica de la tuberculosis en Chile, a pesar del éxito alcanzado en las décadas anteriores, está pasando por una etapa de enlentecimiento que ha impedido que el país se convierta en un área de baja incidencia (tasa menor a 10 casos por 100.000 habitantes $)^{10}$. A la actual velocidad de reducción anual de las tasas de incidencia harían falta 14 años más para lograrlo.
Esta realidad no sólo está presente en Chile, sino en muchos países que han superado la etapa de control y están intentando eliminar la enfermedad, lo que puede deberse a factores que van desde la concentración de los casos de tuberculosis en los grupos más vulnerables, quienes acceden con mayor dificultad a un diagnóstico precoz y tienen peores resultados en el tratamiento, hasta la percepción de la tuberculosis como una patología en disminución que requiere cada vez de mayores recursos para lograr su eliminación ${ }^{11}$.

El nivel central del PROCET ha tenido que realizar una verdadera evangelización sobre la lucha contra la tuberculosis para convencer y reencantar tanto a los niveles directivos como a los equipos de salud de todo el país, de modo de optimizar las actividades de diagnóstico y tratamiento. Aún así, tanto la localización de los casos, como los resultados del tratamiento, todavía están por debajo de las metas establecidas por el programa ${ }^{10}$.

El tema de financiamiento ha sido uno de los grandes desafíos. Múltiples recortes presupuestarios y barreras al flujo expedito de recursos se han convertido en uno de los principales obstáculos a la hora de establecer nuevas estrategias para avanzar hacia la eliminación de la tuberculosis ${ }^{6}$. A esto se agrega la alta rotación de los equipos de salud con sus constantes necesidades de capacitación, y la dificultad del sistema de realizar un verdadero trabajo intersectorial e integrar la participación de la comunidad en su accionar.

Afortunadamente, en el último tiempo surge la esperanza de disponer de mayores recursos para el programa, ya que una cantidad importante de dinero ha sido traspasada a los Servicios de Salud específicamente para ser usados en tuberculosis, y además se está desarrollando un proyecto especial a nivel regional, por parte de la Organización Panamericana de la Salud, para fortalecer los laboratorios supranacionales de tuberculosis, dentro de los cuales se encuentra el Laboratorio de Micobacterias de Referencia de Chile.

\section{Perspectivas a futuro}

Aunque Chile aún no logra convertirse en un país de baja incidencia en tuberculosis, presenta todas las características necesarias para optimizar las acciones del programa y aplicar nuevas estrategias en la lucha contra esta enfermedad, tales como ampliar el accionar sobre el reservorio del Mycobacterium tuberculosis a través de la incorporación de nuevas poblaciones susceptibles de ser sometidas a tratamiento de la infección latente 
o desarrollar proyectos específicos para un diagnóstico precoz, enfocado en grupos de riesgo ${ }^{12}$.

Otra de las estrategias a implementar sería disminuir los tiempos de diagnóstico de la tuberculosis a través del reforzamiento de la red de laboratorios con nuevas tecnologías, desde el aumento en el uso de la microscopía fluorescente para optimizar el diagnóstico por baciloscopia, hasta la incorporación de exámenes moleculares como el GeneXpert, para poblaciones específicas en las que se hace prioritario un diagnóstico rápido, tanto de la enfermedad como de la resistencia a los fármacos ${ }^{13}$.

Pero, lo más importante es optimizar las acciones ya establecidas por el programa: tener equipos bien capacitados a todo nivel, difundir la existencia de la enfermedad y sus síntomas en la población para facilitar una consulta precoz, realizar una búsqueda activa de las fuentes de infección, optimizar el estudio de los contactos y mantener una monitorización estricta de los resultados obtenidos, entre otros ${ }^{14}$.

Finalmente, se hace imprescindible trabajar en conjunto con otros programas de salud que abordan grupos vulnerables, como los programas de VIH, de pueblos originarios, enfermedades cardiovasculares y del adulto mayor, así como avanzar en el trabajo intersectorial para abordar problemáticas sociales y grupos específicos de riesgo de enfermar de tuberculosis.

\section{Reflexiones finales}

A raíz del análisis del Sr. López surgen varias reflexiones sobre el rol de la Salud Pública en la atención médica de Chile. Nuestro país ha sufrido variados vaivenes en su historia sanitaria; desde los planteamientos de "la cuestión social" durante el siglo XIX y parte del siglo XX, hasta las diferentes alternativas surgidas posteriormente con los sucesivos cambios de gobierno y de políticas sanitarias.

Con la creación del Servicio Nacional de Salud el año 1952 se inició la etapa de oro de la Salud Pública en Chile, lo que facilitó el paulatino desarrollo de lo que llegaría a ser un programa de control de la tuberculosis totalmente gratuito a nivel nacional. Sus bases fueron tan fuertes y su trayectoria tan exitosa que las variadas autoridades del Ministerio de Salud que se fueron sucediendo, con distintos enfoques sanitarios, no pudieron menoscabarlo. Hubo que luchar por mantener el concepto de "programa" y de su gratuidad universal; hubo que luchar y frecuentemente perder, para mantener presupuestos razo- nables; hubo que luchar y sufrir con la pérdida o reasignación de funciones de personal valioso y, en fin, hubo que dedicar horas, días y meses en convencer a muchos de lo que era técnicamente obvio, logrando así frenar la tentación de alguna autoridad con poder, de refundarlo todo.

El PCT ha estado inmerso en el debate, que aún no concluye, entre los defensores de los "Derechos Sociales" a través de la Salud Pública promovida por el Estado y los partidarios del neoliberalismo, de las "leyes del mercado" y del creciente rol de la medicina privada en el campo de la salud. Resulta ahora sorprendente que las líneas gruesas del programa hayan sobrevivido a gobiernos tan variados y a visiones de la salud tan diferentes. La fortaleza y perseverancia de un equipo técnico estable a través de los años debe haber contribuido a hacer la diferencia.

Cuando el programa ya había alcanzado un buen grado de efectividad apareció en el mundo un nuevo desafío: la epidemia VIH/SIDA; y cuando ésta ya estaba siendo controlada, estalló la mortífera epidemia de las tuberculosis multirresistentes, en pleno desarrollo actualmente a nivel mundial ${ }^{15}$. En la etapa actual del PROCET el mayor desafío será alcanzar a los llamados "grupos de riesgo", representados especialmente por los individuos más marginales de la sociedad, incluyendo el novedoso desafío que plantea actualmente el creciente número de inmigrantes desde países con una situación epidemiológica más desfavorable ${ }^{16,17}$. Aunque hay que reconocer que la conquista final de la tuberculosis en último término dependerá de un enfoque biosocial que permita alcanzar a los ciudadanos más desposeídos y sus precarios nichos de pobreza ${ }^{18}$.

No cabe duda que la tuberculosis no será eliminada como problema de Salud Pública en Chile sin un mayor compromiso de las autoridades en mantener y reforzar el actual Programa de Control y Eliminación. Es de temer la presente mirada complaciente sobre la situación actual de la tuberculosis en Chile, olvidando que aún fallecen de esta enfermedad más de 200 personas anualmente y que las tasas de descenso de le endemia se han aplanado en los últimos años ${ }^{10}$. La llamada transición epidemiológica, con el progresivo predominio de las enfermedades no trasmisibles, no ha tomado en cuenta que las trasmisibles están lejos de desaparecer. Es de esperar que entre nosotros no se hagan realidad las premoniciones de un experto: "Cuando un programa empieza a ser exitoso, algunos gobiernos, en vez de aumentar los esfuerzos para eliminar la enfermedad, eliminan el programa".

Los más de 40 años que recorre este libro 
estuvieron colmados de intensas actividades no exentas de dificultades, que dan cuenta no sólo de los esfuerzos que se han realizado por controlar la tuberculosis, sino que también reflejan la historia de la salud pública chilena. Vale la pena leer el libro del Sr. López, con sus interesantes descripciones de los avatares del PCT, desde que sólo era un proyecto de programa, pasando por su creación oficial en junio de 1973, hasta las perspectivas actuales de eliminar la enfermedad en un escenario epidemiológico, político y social distinto al que existía medio siglo atrás. Conocer la historia permite aprender del pasado para abordar de mejor forma el futuro.

\section{Bibliografía}

1.- LÓPEZ M. Medicina, política y bien común: 40 años de historia del programa de control de la tuberculosis (1973-2013), Santiago de Chile: Ministerio de Salud; 2015.

2.- FARGA V. Conferencia Héctor Orrego Puelma 2010: I. 80 años de la Sociedad Chilena de Enfermedades Respiratorias: de la tisiología a la neumología, pasando por la medicina interna. Rev Chil Enferm Respir 2011; 27: 31-6.

3.- URZÚA H, JIMÉNEZ DE LA JARA J. Servicio Nacional de Salud: eficaz herramienta de salud social, Medicina Social en Chile. Ediciones Aconcagua; 1977.

4.- FARGA V, CARRASCO E, YÁÑEZ A, MENA G, BUSEL I, VALENZUELA P, et al. Algunas novedades en el tratamiento de la tuberculosis. Boletín Hospital San Juan de Dios 1965; 12: 404.

5.- HERRERA L. Estado actual de la microbiología de la tuberculosis en Latinoamérica. Boletín de la Oficina Sanitaria Panamericana 1970; 208.

6.- ZÚÑIGA M. La eliminación de la tuberculosis como un problema de salud Pública. Situación de Chile en el año
2008. Rev Chil Enferm Respir 2009; 25: 117-26.

7.- FARGA V, VALENZUELA P, MENDOZA F, TORRES I, ICEKSON I. Quimioterapia de corta duración. Estudios operacionales controlados de Chile. Bol Unión Intern Tuberc 1983; 58: 102-7.

8.- FARGA V, CAMINERO J. Tuberculosis. Tercera Edición. Editorial Mediterráneo. 2011.

9.- STYBLO K. Evaluación y recomendaciones del Programa de Control de la Tuberculosis de Chile. Rev Chil Enferm Respir 1998; 14: 104-15.

10.- Programa Nacional de Control y Eliminación de la Tuberculosis. Informe de situación Chile 2014. Ministerio de Salud. 2015.

11.- PEÑA C. El difícil camino del control sanitario de la tuberculosis. Rev Chil Enferm Respir 2012; 28: 311-6.

12.- HERRERA T. Tuberculosis en Chile: ¿Podemos retomar el camino hacia la eliminación? Rev Chil Enferm Respir 2014; 30: 225-9.

13.- VALLEJO P, RODRÍGUEZ J, SEARLE A, FARGA V. Ensayo Xpert MTB/RIF en el diagnóstico de tuberculosis. Rev Chil Enferm Respir 2015; 31: 127-31.

14.- HERRERA T. La situación de la tuberculosis en Chile y los actuales desafíos. Visita de la OPS al programa de control de la tuberculosis de Chile. Rev Chil Enferm Respir 2013; 29: 46-9.

15.- RODRÍGUEZ J, IRAZOQUI P. Guías para el manejo de la tuberculosis resistente. Organización Mundial de la Salud 2011. Rev Chil Enferm Respir 2012; 28: 153-8.

16.- HERRERA T. Grupos de riesgo para tuberculosis en Chile. Rev Chil Enferm Respir 2015; 32: 15-8.

17.- YÁÑEZ A. Tuberculosis en inmigrantes. Situación Chile-Perú. Rev Chil Enferm Respir 2010; 26: 164-5.

18.- ORTBLAD K, SALOMON J, BARNIGHAUSEN T, ATUN R. How to eliminate tuberculosis. Stopping tuberculosis: a biosocial model for sustainable development. Lancet 2015. doi: 10.1016/S0140-6736(15)003244 Disponible en http://www.thelancet.com/series/howto-eliminate-tuberculosis.
Correspondencia a:

Dra. Tania Herrera M.

Directora, Programa de Control y

Eliminación de la Tuberculosis (PROCET)

Ministerio de Salud. Santiago. Chile.

Email: tania.herrera@minsal.cl 